\title{
AC temperature estimation of power electronic devices using 1D thermal modeling and IR thermography measurements
}

\author{
by M. Kopeć, B. Więcek
}

\begin{abstract}
Lodz University of Technology, 211/215, Wolczańska Str., 93-005, Łódź, Poland, michal.kopec@edu.p.lodz.pl,
\end{abstract} boguslaw.wiecek@p.lodz.pl

\begin{abstract}
This paper presents the novel method and program for reconstruction of the thermal impedance in the heat source based on the IR radiation measurement on the surface of an electronic device. In this approach, temperature is measured after applying power pulse to the element. The thermal modelling of an electronic device is used. Automatic synchronization of temperature measurement and power excitation is available in developed controller. The results from the program IRTherm are compare with the results obtained from the Finite Element Method in a commercial simulation software. Heat transfer is modelled using thermal-electrical analogy by Foster and Cauer networks consisting of thermal resistances $R_{\text {th }}$ and capacitances $C_{t h}$. The results are presented as the thermal impedance in the frequency domain (the Nyquist plot) and thermal time constant distribution.
\end{abstract}

\section{Introduction}

The transient thermal modelling is now very important for various field of science and technology. It is used in many practical applications [1-4]. Different methods are available for thermal characterization of multilayer structures. Modelling using Finite Element Method (FEM) is very popular for solving thermal problem in the time domain. The main requirement of FEM method is the high computational power. Having temperature response and power excitation, the inverse thermal modelling leads to the identification of a thermal multilayer system. There are various method available for system identification, such as the Network Identification by Deconvolution (NID), the Continuous-time System Identification (CONTSID), the Vector Fitting (VF), the Computer-Aided Program for Time-series Analysis and Identification of Noisy Systems (CAPTAIN) and Transfer Function Estimation (TFEST) implemented in MATLAB environment [5-20].

In this paper one shows a novel software tool for the reconstruction of the thermal impedance in the heat source of the tested object based on the IR radiation measurement. It calculates Nyquist plot of thermal impedance in the frequency domain and thermal time constants spectrum.

\section{1D analytical and numerical models}

1D analytical thermal model of a transistor is based on the 6-layers structure consisting of the epoxy enclosure, the silicon transistor layer, the cooper substrate, the thermal interface layer (thermally conductive grease), the heat sink transferring heat to the ambient and substrate as presented in Figure 1. The substrate layer is place where the heat sink is in the contact with the base (holder for tested object). Adding $n_{6}$ layer simplified temperature modelling in the steadystate.

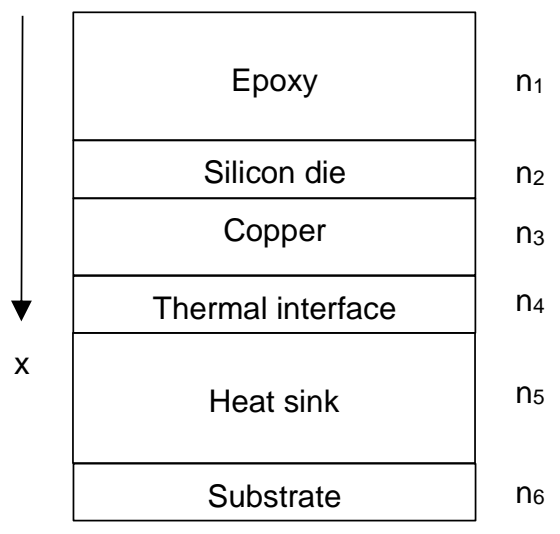

Figure 1. $1 D$ analytical thermal model 
For each layer is described by the Fourier-Kirchhoff heat transfer equation (1)

$$
k_{i} \frac{\partial^{2} T(x, t)}{\partial x^{2}}-c_{v i} \frac{\partial T(x, t)}{\partial t}=-q_{v i}
$$

where $k_{i}$ - thermal conductivity of $i$-th layer $(\mathrm{W} / \mathrm{m} \cdot \mathrm{K}), c_{v i}=\rho_{i} c_{p i}-$ specific heat per volume unit $\left(\mathrm{J} / \mathrm{m}^{3} \mathrm{~K}\right)$.

The analytical model is presented in frequency domain (2) by using Laplace transform, for $s=j \omega$ [22].

$$
L_{i}^{2}(j \omega) \frac{\partial^{2} T(j \omega)}{\partial x^{2}}-T(j \omega)=-\frac{L_{i}^{2}(j \omega)}{k_{i}} q_{v i}
$$

where power density is $q_{v i}\left(\mathrm{~W} / \mathrm{m}^{3}\right)$ and diffusion length $L_{i}(\mathrm{~m})$ for $i$-th layer is expressed as:

$$
L_{i}(j \omega)=\sqrt{\frac{k_{i}}{j \omega c_{v i}}}
$$

Analytical solution of (2) in the Laplace domain for $i$-th layer can be presented as:

$$
T(x, j \omega)=A_{i} e^{-\frac{x}{L_{i}}}+B_{i} e^{\frac{x}{L_{i}}}+\frac{L_{i}^{2}}{k_{i}} q_{v i}
$$

Flux continuity at the front and bottom surfaces (convective cooling) are defined as in (5) and (6).

$$
\begin{gathered}
-k_{1} \frac{d T_{1}(0)}{d x}=h_{\text {front }} T_{1}(0) \\
-k_{5} \frac{d T_{1}(0)}{d x}=h_{\text {bottom }} T_{6}\left(d_{6}\right)
\end{gathered}
$$

where $d_{6}$ is the thickness of the 6 -th layer (substrate).

Temperature is then calculated by solving the set linear equations formulated by the 6 relations (4) with temperature in each layer as the unknown variable.

At last, the thermal impedance is calculated using temperature in a power device and takes the form of Nyquist plot as:

$$
Z_{t h}(x, j \omega)=\frac{T_{2}(x, j \omega)}{P(j \omega)}
$$

where $T_{2}$ is the mean temperature in the silicon layer.

In order to confirm the correctness of the analytical model, the alternative 1D numerical FEM model was elaborated to get temperature in the transistor's junction [23]. The resulting mesh gives 213 degrees of freedom. It is a very time-consuming task and it lasts about 200 minutes. The FEM model was done on Core i7-9750H with 24GB RAM and NVMe SSD disc. In comparison with the analytical model implemented in MATAB, the run time of the simulation is a few seconds.

The materials parameters of the transistor structure layers chosen for the simulations are presented in Table 1.

Table 1.The material properties of transistor structure used for modelling

\begin{tabular}{|c|c|c|c|c|c|}
\hline Layer no. & Layer & Thickness $(\mathrm{m})$ & $\begin{array}{c}\text { Thermal } \\
\text { conductivity } \\
\left(\mathrm{Wm}^{-1} \mathrm{~K}^{-1}\right)\end{array}$ & Density $\left(\mathrm{kg} / \mathrm{m}^{3}\right)$ & $\begin{array}{c}\text { Heat capacity } \\
\left(\mathrm{J} \cdot \mathrm{kg}^{-1} \mathrm{~K}^{-1}\right)\end{array}$ \\
\hline $\mathrm{n}_{1}$ & case & $3.1 \mathrm{e}-3$ & 1.2 & 950 & 1000 \\
\hline $\mathrm{n}_{2}$ & die & $300 \mathrm{e}-6$ & 148 & 2330 & 720 \\
\hline $\mathrm{n}_{3}$ & metal substrate & $1.275 \mathrm{e}-3$ & 237 & 2700 & 950 \\
\hline $\mathrm{n}_{4}$ & thermal interface (grease) & $0.4 \mathrm{e}-3$ & 4 & 2500 & 800 \\
\hline $\mathrm{n}_{5}$ & heat sink & $4.5 \mathrm{e}-3$ & 237 & 2800 & 950 \\
\hline $\mathrm{n}_{6}$ & substrate & $0.1 \mathrm{e}-3$ & 58 & 7900 & 500 \\
\hline
\end{tabular}




\section{IRTherm program for estimation thermal impedance of power electronic devices}

In this research, the novel IRTherm program is developed in the MATLAB environment. The IRTherm program is used to analytical modelling of the multilayer structure. This program has the graphical user interface to present data in graphical or text format for further analysis, as shown in Figure 2. The program layout is user friendly, the functions are activated by buttons and results are shown graphically by plotting curves or numerically in tables.

The exemplary research were performed for NPN bipolar power transistor BD911 with the heat sink. The analysis starts with registering temperature in time by measuring IR radiation using the IRTherm program (Measurement - blue line in Figure 2) The radiation measurement data are registered using the developed controller [21].

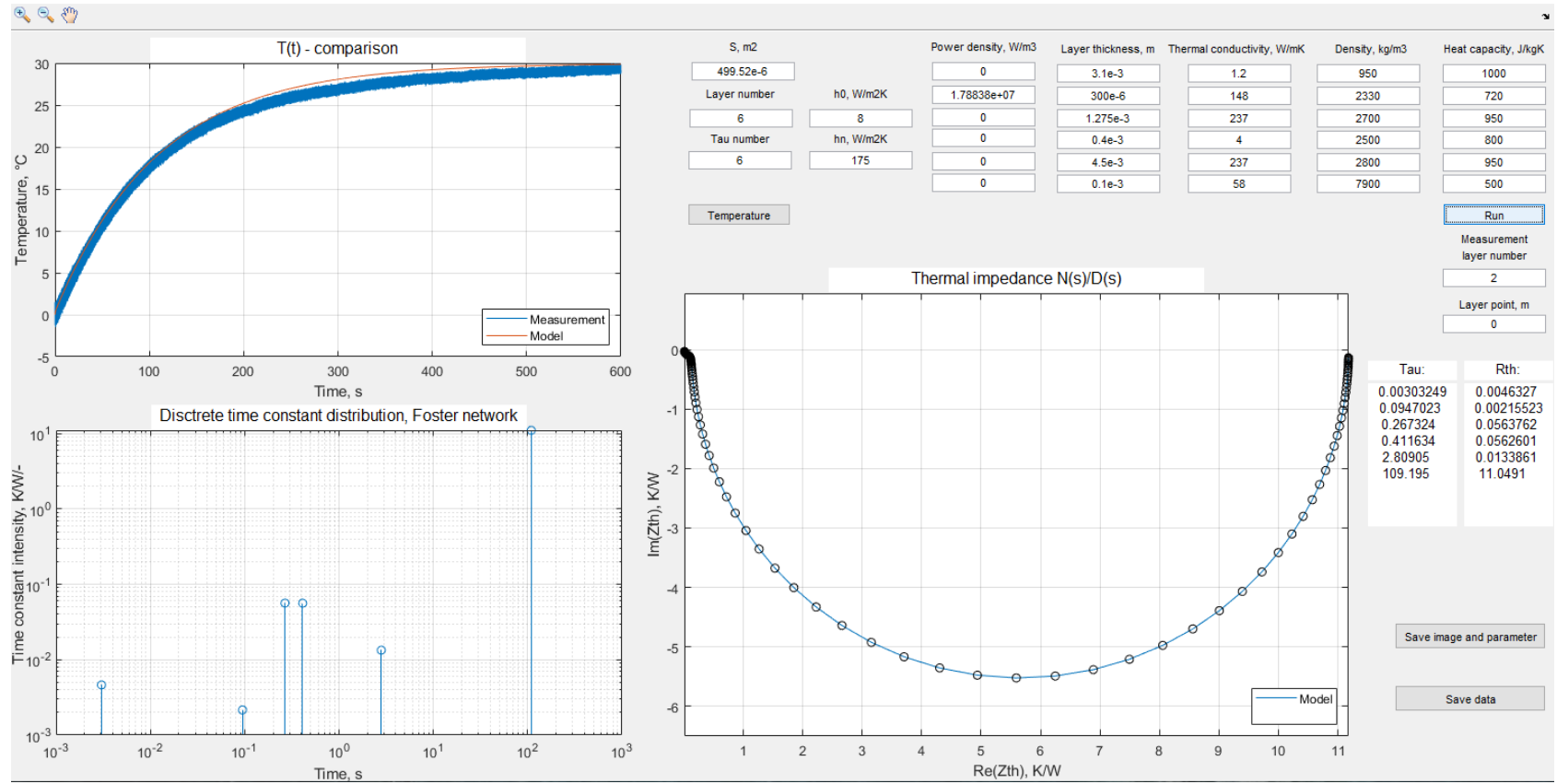

Figure 2. IRTherm program main window in Matlab environment

Next step is matching geometrical and thermophysical data in model so that modelled temperature curve coincides with the radiation measurement. The used data are shown in Table 1.

After the measurement, the models are tuned to fit the simulated and measured temperature curves on the outer surface of the tested element. Then, the layer number $n$ in the models is changed to die layer $\left(n_{2}=2\right)$. The parameter named Layer point is set to the middle point of the silicon die. The user can set values from 0 to $300 \mu \mathrm{m}$ to enable observation at any point in die layer. Finally, the function Run can calculate temperature in the heat source as it is presented in Figure 2 brown line. Finally, the program calculates Nyquist plot of thermal impedance in frequency domain and thermal time constant spectrum.

\section{Results}

The obtained results are the temperature curves (Figure 3,4), the thermal impedance of Nyquist plots $($ Figure 7,8$)$ and time constant distributions (Table 2). The temperature curves are shown on the outer side of transistor's case (Figure 3,4 ), and in the transistor junction (Figure 5,6). 
10.21611/qirt.2020.161

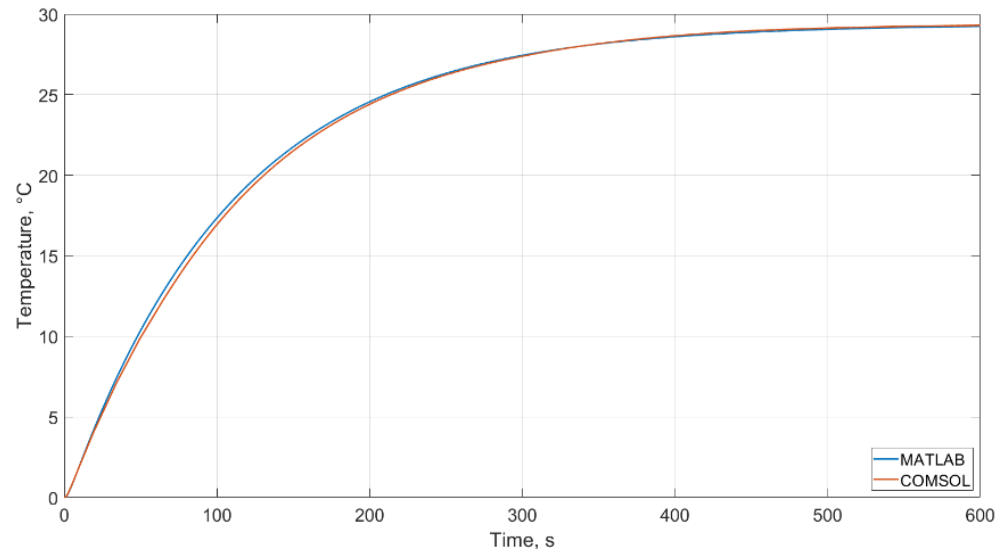

Figure 3. Temperature on the outer side of the transistor's case: blue line - simulation result from MATLAB, brown line - simulation result from COMSOL

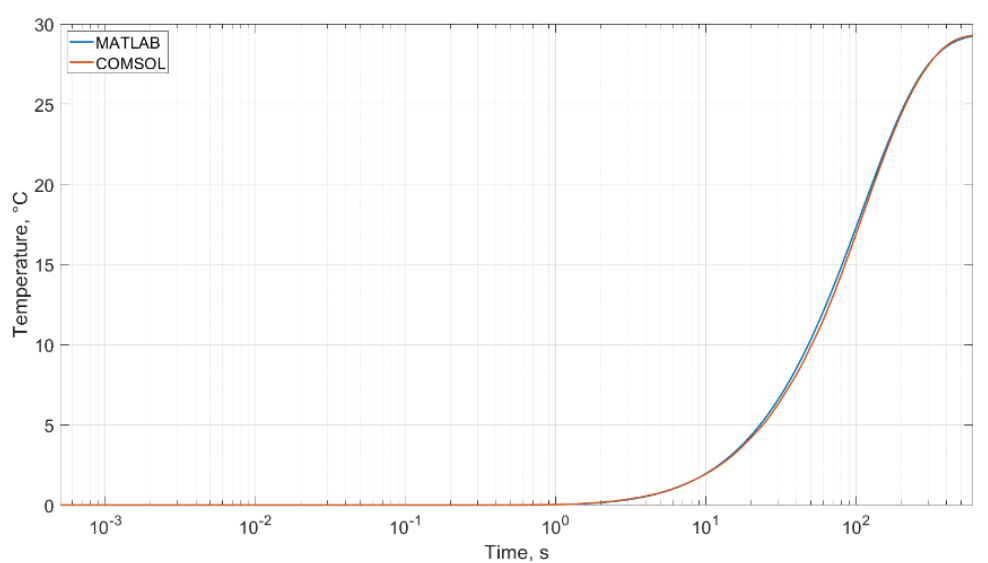

Figure 4. Temperature on the outer side of the transistor's case in logarithmic scale: blue line - simulation result from MATLAB, brown line - simulation result from COMSOL

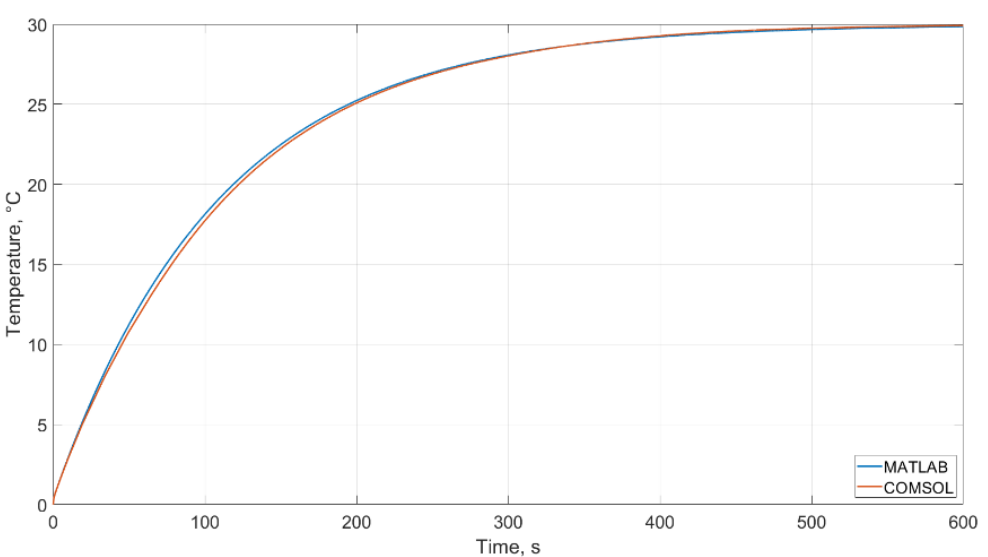

Figure 5. Temperature in the transistor junction: blue line - simulation result from MATLAB, brown linesimulation result from COMSOL 


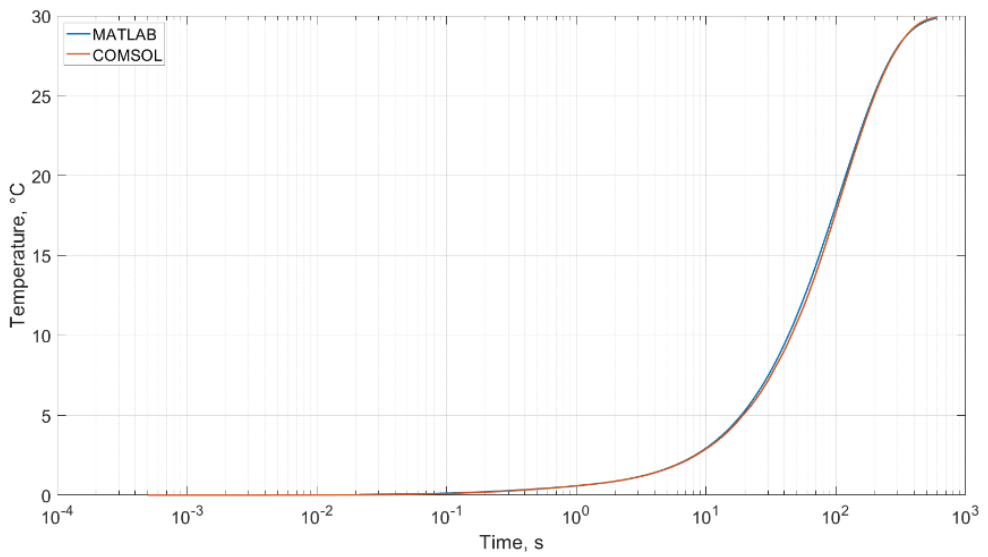

Figure 6. Temperature in logarithmic scale in the transistor junction: blue line - simulation result from MATLAB, brown line - simulation result from COMSOL

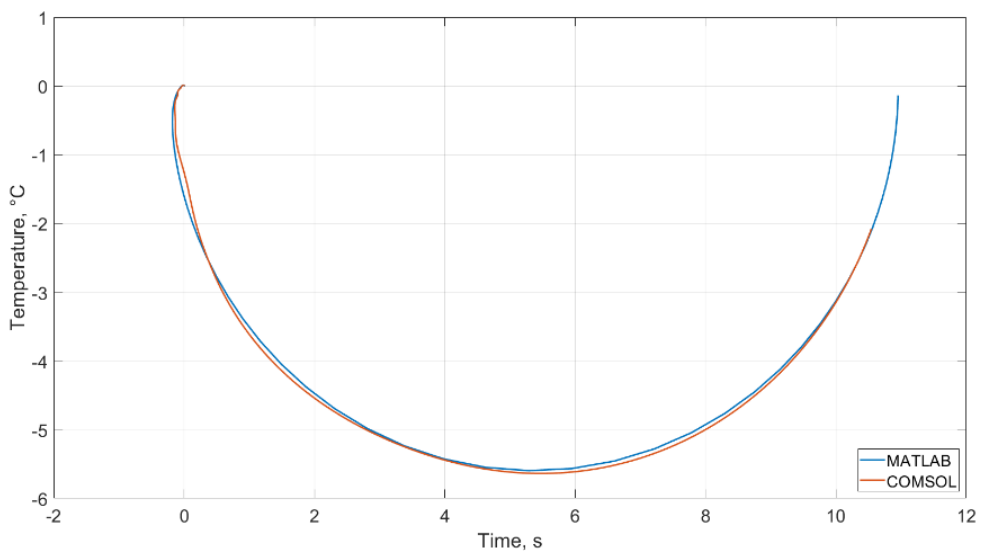

Figure 7. Nyquist plot on the outer side of the transistor's case: blue line-simulation result from MATLAB, brown line - simulation result from COMSOL

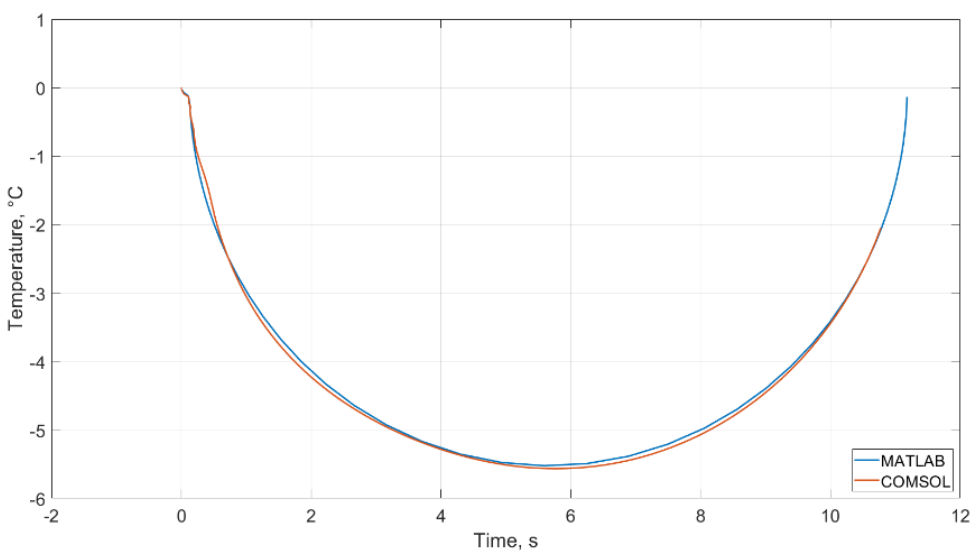

Figure 8. Nyquist plot in the transistor junction: blue line - simulation result from MATLAB, brown linesimulation result from COMSOL

Table 2. Values of thermal time constants and thermal resistances in the die

\begin{tabular}{|c|c|c|c|}
\cline { 2 - 4 } \multicolumn{1}{c|}{} & \multicolumn{2}{|c|}{ IRTherm } & COMSOL \\
\hline No. & $\tau,(\mathrm{s})$ & $\mathrm{R}_{\mathrm{th}},(\mathrm{K} / \mathrm{W})$ & $\tau,(\mathrm{s})$ \\
\hline 1 & 0.0030 & 0.0046 & 0.001412 \\
\hline 2 & 0.0947 & 0.0022 & 0.008318 \\
\hline 3 & 0.2673 & 0.0564 & 0.40738 \\
\hline 4 & 0.4116 & 0.0563 & 109.6500 \\
\hline 5 & 2.8090 & 0.0134 & \\
\hline 6 & 109.1950 & 11.0491 & \\
\hline
\end{tabular}




\section{Discussion and conclusions}

The comparison of analytical and FEM models for inverse thermal modelling of power electronic devices is shown in this paper. Both models are built with the same geometrical, thermophysical parameters and boundary conditions. However, the method of calculating the results is different. Also, computation times are different. The analytical model in IRTherm program is very fast compared to FEM model. The thermal impedance from FEM method is calculated using commercially available software for thermal object characterization based on Network Identification by Deconvolution (NID) technique [5]. In turn, the thermal impedance from IRTherm is calculated using Transfer Function Estimation (TFEST) implemented in MATLAB. It can be noticed that temperature curves on the outer enclosure surface of the transistor's case (Figure 3,4) and in the junction (Figure 5,6) coincide with each other. The temperature curves are very good matched also at the beginning. It agrees very well with high angular frequency part in the Nyquist plot (Figure 7, 8). Referring to time constant values, it can be concluded that one thermal time constant is significant in both models, i.e. the longest time constant $\tau_{6}$. The amplitude values of thermal time constants have significant information's. The high value of amplitude means that component in the equation of the temperature approximation have significant contribution. Moreover, it influences the transient temperature curve more. The longest thermal time constant is associated with low angular frequency. It depends mainly from convection. Shorter thermal time constant related to construction of the tested object. Finally, it can be concluded that two thermal time constants $\tau_{4}$ and $\tau_{6}$ may be considered for quantitative analysis. The thermal time constants $\tau_{1}, \tau_{2}, \tau_{3}$ and $\tau_{5}$ have small amplitude values, so contribution in temperature approximation is insignificant. The thermal time constant $\tau_{3}$ should be also discussed. The new method based on TFEST function calculated its value. The NID technique is used to thermal impedance from FEM model. As a result $\tau_{3}$ is not calculated. The reason is another way of calculating thermal time constant $\mathrm{T}$ in both models. In NID technique continuous thermal time constant distribution is calculated, while using TFEST function one can obtain the discrete form of this distribution.

\section{REFERENCES}

[1] Chatziathanasiou V., Chatzipanagiotou P., Papagiannopoulos I., De Mey G., Więcek B., "Dynamic thermal analysis of underground medium power cables using thermal impedance, time constant distribution and structure function". Applied Thermal Engineering vol. 60(1-2), pp. 256-260, 2013.

[2] Chatzipanagiotou P., Chatziathanasiou V., De Mey G., Więcek B., "Influence of soil humidity on the thermal impedance, time constant and structure function of underground cables: a laboratory experiment. Applied Thermal Engineering 113, pp. 1444-1451, 2017.

[3] Chatzipanagiotou P., Strąkowska M., De Mey G., Chatziathanasiou V., Więcek B., Kopeć, M. "A new software tool for transient thermal analysis based on fast IR camera temperature measurement". Measurement Automation Monitoring vol. 63, 2017.

[4] Vermeersch B., "Thermal AC modelling, simulation and experimental analysis of microelectronic structures including nanoscale and high-speed effects". Ph.D. thesis, Ghent University, Faculty of Engineering, 2009.

Buttner W. "Ein numerisches Verfahren zur Exponential approximation von transienten Wärmewiderständen". Archiv Elektrotech, vol. 59, no. 6, pp. 351-359, 1977.

[5] Corporation M.G, "T3Ster-Master Thermal Evaluation Tool". Mentor Graphics Corporation, The address of the publisher, version 2.2

[6] Drmac Z., Gugercin S., Beattie C. "Quadrature-based vector fitting for discretized H2 approximation. SIAM Journal of Scientific Computing, vol. 37, no. 2, pp. A625-A652, 2015.

[7] Garnier H., Mensler M., Richard A. "Continuous-time model identification from sampled data: implementation issues and performance evaluation”. International Journal of Control, vol. 76, no. 13, pp. 1337-1357, 2003.

[8] Górecki K., Rogalska M., Zarębski, J. "Parameter estimation of the electrothermal model of the ferromagnetic core". Microelectronic Reliability, vol 54, no. 5, pp. 978-984, 2014.

[9] Górecki K., Zarębski J. "The influence of the selected factors on transient thermal impedance of semiconductor devices. In: 2014 Proceedings of the 21st International Conference on Mixed Design of Integrated Circuits \& Systems (MIXDES), pp. 309-314. IEEE 2014.

[10] Hellen E.H. "Padé-laplace analysis of signal averaged voltage decays obtained from a simple circuit". American Journal of Physics, vol. 73, no. 9, pp. 871-875, 2005.

[11] Kałuża M., Więcek, B., De Mey G., Hatzopoulos A., Chatziathanasiou V. "Thermal impedance measurement of integrated inductors on bulk silicon substrate. Microelectronic Reliability, vol. 73, pp. 54-59, 2017.

[12] Jakopovid Z., Bencic Z., Koncar R. "Identification of thermal equivalent-circuit parameters for semiconductors. In: 1990 IEEE Workshop on Computers in Power Electronics, pp. 251-260. IEEE 1990.

[13] Jibia A.U., Salami M.J.E. "An appraisal of Gardner transform-based methods of transient multiexponential signal analysis. International Journal of Computer Theory and Engineering, vol. 4, no.1, 2012.

[14] Ljung L. "Experiments with Identification of Continuous Time Models". Linköping University Electronic Press, 2009.

[15] Marco S., Palacın J., Samitier J. "Improved multiexponential transient spectroscopy by iterative deconvolution. IEEE Transactions of Instrumentaton and Measurement, vol. 50, no. 3, pp. 774-780, 2001. 
[16] Mathworks: Transfer function estimation (tfest) help. https://nl.mathworks.com/help/ident/ref/tfest.html. Accessed 01 July 2020.

[17] Ozdemir A.A., Gumussoy S. "Transfer function estimation in system identification toolbox via vector fitting". IFACPapersOnLine, vol. 50, no. 1, pp. 6232-6237, 2017.

[18] Young P., Jakeman A. "Refined instrumental variable methods of recursive time series analysis part iii. extensions". International Journal of Control, vol. 31, no. 4, pp. 741-764, 1980.

[19] Szekely V., "On the representation of infinite-length distributed RC oneports". IEEE Trans. Circuits Syst., vol. 38, pp. 711-719, 1991.

[20] Szekely V., "Identification of RC networks by deconvolution: Chances and limits". IEEE Trans. Circuits Syst., vol. 45, no. 3, pp. 244-258, 1998.

[21] Kopeć M., Wiecek B. "Thermal characterization of electronic components using single-detector IR measurement and 3D heat transfer modelling". 7th International Conference Mixed Design of Integrated Circuits and Systems MIXDES 2020, Wroclaw, Poland, June 25-27, 2020.

[22] Strąkowska M., Więcek B. "Thermal modeling of planar and cylindrical biomedical multilayers structures in frequency domain", Measurement Automation Monitoring, no. 02, pp. 32-36, 2019.

[23] https://www.comsol.com 